\title{
Establishment of Comprehensive Evaluation System of Fundamental Chemistry Experiment Course
}

\author{
Boquan Jiang, ${ }^{*}$ Ting Liu,Min Xu,Xu Li, ,Zhengping Chen \\ Department of Biology and Chemistry \\ Institute of Science \\ Nanchang University College of Science and Technology, \\ Nanchang, China \\ * corresponding author. email: jbq_win@163.com. , mobile phone number :13397080986
}

\begin{abstract}
The reform of fundamental chemistry experiment (FCE) course is one of the important gateway to cultivate creative talents. Establishing a scientific comprehensive evaluation system of FCE course is one of the important contents of the reform of FCE course. In order to scientifically, reasonably, objectively and fairly evaluate the teaching quality of FCE course, an operational evaluation system is built in this paper. This system involves the grade of ordinary times, the grade of theory examination, the grade of operation examination and the grade of open experiment examination.According to the system, the evaluation is carried out throughout the experimental teaching process, which not only promote the students' theoretical study, but also stimulate their enthusiasms of autonomous learning and increase their practical beginning abilities.
\end{abstract}

Keywords- fundamental chemistry experiment; evaluation; establishing; teaching reform

\section{I . INTRODUCTION}

The Fundamental chemistry experiment (FCE) course, including inorganic chemistry,organic chemistry, physical chemistry and analytic chemistry experiment, is an independently opened compulsory basic course in universities. The FCE course is opened for many specialities of science and technology. The goals of the FCE course mainly are to enable the students to master the general knowledge, basic experimental operation skills, independent experimental ability and correct learning method of combining theory with practice, increase the students' independent thinking and working abilities, develop the students' scientific working attitude and cultivate the students' innovation consciousness and ability[1-5].To well learn the FCE course at their first year will lay a solid foundation for their subsequent practical links, such as specialized experiment and graduation thesis. The students can learn various of chemical unit operation in the basic chemistry laboratory , such as fluid conveying, sedimentation, filtration, stirring, heat transfer, evaporation, absorption, distillation, rectification, extraction, drying,etc. which is greatly helpful to learn the following courses of principle of chemical engineering and chemical engineering experiment, which are the important professional foundation courses. In our college, inorganic experiment course and analytic experiment course are combined into university chemistry experiment (UCE) course and the FCE course is opened to the majors of applied chemistry, biologic engineering, biologic technology, pharmaceutical engineering, polymer materials and engineering, environmental engineering and water supply and drainage. The purposes of the FCE course are for the students to master the basic operations and skills of chemistry experiment and general preparation and purification methods of compounds. The FCE course is a comprehensive course with the collection of inorganic chemistry experiment, organic chemistry experiment and the analytic chemistry experiment in the original curriculum system. The FCE course contains 25 experiments with 4 credits and 120 hours for the majors of pharmaceutical engineering, polymer materials and engineering, 20 experiments with 3 credits and 90 hours for the majors of biologic engineering and biologic technology in our college.The course grade is comprehensively evaluated. In order to increase the teaching quality of the FCE course, a set of scientific, reasonable and operational comprehensive evaluation system of FCE course has been established in recent years and being smoothly carried out. The contents of the system involve the grade of ordinary times, the grade of the theoretical examination, the grade of the operation examination and the grade of open experiment examination.

\section{GRADE OF ORDINARY TIMES}

The assessment of the grade of ordinary times is mainly to inspect the students' understanding of the purpose, principle and method of each experiment, beginning ability, ability to analyze and solve practical problems during the experiment, ability to treat the experimental data and results, ideological quality or experimental habits and the quality of the written experiment report[6-10]. The grade of ordinary times is $60 \%$ of the total grade. The grade at ordinary times includes four parts: preview, experimental operation, experimental attitude and writing experimental report. The preview requires the students to carefully prepare experiment content concerning with the understanding of the purpose, principle and operation steps 
of the experiment, search the physical and chemical properties of the demanded chemicals, clearly realizing the key and attention items of the experiment, writing the preview report for checking by the teachers and listen to the teacher's instructional explanation and answer the teacher's questions. The experimental operation requires the students to independently finish their experimental target; operate with specification; focus on analyzing and solving the problems encounted during the experiment; timely and faithfully record the observations and the original experimental dada. If some students failure to the experiment due to their wrong operations or other special causes, they will be asked to redo the experiments. The experimental attitudes require the students to obey the laboratory regulations and rules; listen to the teacher' guidance; respect for the teachers and laboratory staff; take care of the equipment and instrument; pay attention to the energy conservation and emission reduction, safety and sanitation. The experimental report requires the students to write down the purpose and principle of the experiment with their own words and do not completely copy the textbook; list the main chemicals, equipment and instruments; clearly draw up the experimental device; give the certain chemical and physical properties of chemicals related to the experiment by searching; make out the experimental steps and describe the observed experimental phenomena of each step; record the use levels of raw materials and chemicals and the original data (such as the output of product, solution volume consumed in acid-base titration,etc.) obtained from the experiment, which should be checked by the teacher on the spot before leaving the laboratory; calculate the experimental target values; analyze and discuss the experimental results. The grade of preview, experimental operation, experimental attitude and writing experimental report are 10\% (10 marks), 25\% (25marks), $10 \%$ (10marks)and $15 \%$ (15marks) of the total grade, respectively.

\section{GRADE OF THEORY EXAMINATION}

Experiment is a process of combining theory with practice. In the process, the theory is used to guide the experiment and, in turn, it is verified through the experiment [11-12]. It is impossible for the students to successfully carry out an experiment without theoretical instruction. In the first two semesters of university, the theoretical course and experimental course of basic chemistry are independently opened to the fresh-students at same time.We require the students to organically combine the theoretical course with experimental course of the basic chemistry, for example, using the theories of "the error in quantitative analysis", "data treatment of analytic results" and "effective numbers and operation rules" learned from the theoretical course of university chemistry to guide the data treatment of the experiments; using the theories of "acid-base titration method","REDOX titration method" and "coordination titration method" learned from the theoretical course of university chemistry to guide the university chemistry experiments of "The calibration of hydrochloric acid concentration","The preparation and calibration of EDTA standard solution"and the other experiments related to quantitative analysis; using the theories of "The classification of organic compounds", "The classification of organic reactions" and "The classification of organic reaction mechanism” learned from the theoretical course of organic chemistry to guide the organic chemistry experiments of "The synthesis of diphenyl carbinol", "The preparation of ethyl acetate system" and "The synthesis of amino acid". In order that the students can make a close combination between theory and practice and consolidate their theoretical knowledges through experiments, we established the "collection of questions and answers" including the purpose and principle of each experiment and the "thinking questions" of each experiment. The purpose of theory examination is to check whether the students have firmly grasp the theories on the experiments or not. Before the theory examination, the students are asked to review all the experiments they have done and understand all the questions in the "collection of questions and answers". The test questions are prepared in the form of "note question" by the teachers. During the examination, the students firstly extract 5 of note questions and then give they answers by oral or writing on the spot in a limited amount of time. The grade of the theory examination is $10 \%$ of the total grades (10 marks). Each question is assigned to 2 marks.

\section{IV.GRADE OF OPERATION EXAMINATION}

The chemical experiment operation standardization is the guarantee of experimental success and safety. Firstly, whether the chemical experimental operation is standardized or not directly affects the accuracy of the experimental results and relates to the success or failure of the experiments. During the university chemistry experiments, even a small non-standard operations may cause the large error in the data. The incorrect data can not be used or may cause a wrong result. For example, the non standard operation may result in an incorrect judgment of titration end point which makes a wrong concentration of the calibrated standard solution during the acid-base titration process. The non-standard operation may cause product loss during the vacuum or suction filtration. The non-standard operation may not produce the expected experiment phenomena during the properties experiments, which causes a wrong judgement and lead to a failed experiment. Secondly, many kind of chemicals used in the experiments are poisonous and harmful, some poisonous and harmful substances may produced in the experiments. and there exist many factors causing burning and explosion. The nonstandard operations may make the poisonous and harmful substances damage the operators and surrounding environment. Some of non-standard operations may directly 
trigger the explosion and fire accidents, which not only injures the operators but also damage the goods. Thirdly, the non-standard operations may damage the precious precision instruments, causing great economic losses. In the experiments, the teachers repeatedly stress the importances of the standard operations and require the students to do standard operations. In order to exame the student's abilities of mastering basic operation skills, the operation examination is carried out at the end of the course. The operation examination can be conducted by that the teachers prepare the note type questions in advance and then the students temporarily extract the questions and show their experimental performances on the scene. The grade of operation examination is $10 \%$ of the total grade (10 marks).

\section{GRADE OF OPEN EXPERIMENT EXAMINATION}

Open teaching mode of FCE course is an important channel to cultivate the creative talents. In order to increase the student's independent design, application and innovation ability to reflect the education idea of "with the students as main body", it is imperative to build an open teaching mode of FCE course [16-20]. The open experimental teaching requires the teachers to set experiment projects, involving applied, design, comprehensive, research and innovation types of experiment. Comprehensive and design type of experiment is more suitable to the university fresh students. The open experimental teaching requires the students to freely select the project of comprehensive and design experiments set by the teachers and independently finish their whole experiment process through their own thinking, exploration and operation under the teachers' instruction. This mode is the important gateway to develop the student's potentials, increase the student's scientific quality and cultivate the student's innovation ability. At present, the main contents of FCE course are compulsory and verified projects in our college. The laboratories are only opened to student's professional experiment, graduation thesis and extracurricular scientific and technologic activities. Therefore, it is urgent for us to implement open teaching of FCE course.The teachers have set 8 comprehensive and design experiment projects for both UCE course and organic chemistry experiment (OCE) course. In this semester, the open teaching of OCE course is being conducted to the students. During the open experiment process, the students are required to freely select the experiment project and independently complete their project by taking various of steps, such as independent literature review, making design scheme, predicting required chemicals and instruments, establishing experimental device, starting work, data treatment, writing experimental report, reporting the experiment by PPT file, etc.. Mutual discussions and exchanges between the students and between the students and teachers are advocated. The laboratories provide the experimental place, time and instruments \& chemicals for the students to ensure the open experiment smoothly conducted. The orderly and standardized experiments can effectively promote the student's abilities of autonomous learning, cooperative learning and creative learning. The evaluated factors of open experiment involve the students' abilities of literature review, scheme design, standard operation, experimental results, and experimental report and open reply with PPT file. The grade of open experiment examination is $20 \%$ of the total grades and each factor is assigned to 4 marks.

\section{ACKNOWLEDGMENT}

This subject comes from " the Project on Demonstration Laboratory Construction " financially supported by Nanchang University College of Science and Technology.

\section{REFERENCES}

[1] Liu Kui, Wang Jianmin and Sun Hua. “ Exploration and practice of establishing a comprehensive evaluation system”. Gao Xiao Shiyanshi Gongzuo Guanli, 2010, Vol.104, issue 2, pp. 32-33,77.

[2] Li Changjian, Xie yimin. “Talking about the laboratory management system reform of newly built undergraduate course colleges and universities”. Forum on Contemporary Education, 2006, issue 11, pp. 85-86.

[3] Cao Aiwen, Jiang Liwen. “ The reform and practice of university laboratory management system”. Journal of East China Jiaotong University, 2005, issue12, pp.118-119.

[4] Wang Guirong, Yang Gengshe.“ Research of university laboratory management system reform based on the innovation ducation idea”.Technology and Innovation Management, 2008, issue 6, pp. 559-561.

[5] Chen Huiyang, Yu Tuyuan.“ The reform and practice of laboratory management system”. Review of Journal of Zhongshan university, 2005, issue 1, pp. 151-154.

[6] Su Zhi. “ Design of multimedia courseware for chemistry experiment operating ”. .Journal of XinJiang Normal University, 2002, Vol.21, issue1, pp. 38-41.

[7] Zhu Hongtao,Quan Yuheng. “ The importance of chemical experiment standardized operation ”. Science and Technology Innovation Herald, 2012, issue 13, pp. 251.

[7] Hao Zhengfang. "Discussing the importance of preparing lessons before experiment to improve the quality of experiment teaching ". Journal of Shijiazhuang Vocational Technology Institute, 2003, Vol. 5, issue 4, pp. 59-60.

[8] Jiang Lingfeng, Liao Hailin. " Strengthening the inorganic chemical experiment basic operation specification in order to improve the teaching quality”. Light Industry Science and Technology, 2014, Vol. 183, issue2, pp. 135-136.

[9] Tie Burong. "Inorganic Chemistry Experiment” [M]. .Beijing:China press of traditional Chinese medicine, 2012. 
[10] Su Fang, Yuan Zhuangdong • “.The optimization discussions of the preparation schemes of inorganic chemical experiments” . .Journal of Jining University, 2009, Vol.30, issue 6, pp. 43-45.

[11] Li Hongmei, Lu Jiang and Wang Xianfeng. “ Practice and summary of college basic chemical experiment teaching ”. University Chemistry, 2011, Vol. 26, issue 5, pp. 39-42.

[12] Wang Xueying, Xu Xiaoxing and Li Qiaoyun. “ The construction of system of basic chemical experimental teaching and evaluation based on the applied talents training “. .Journal of Changshu Institute of Technology, 2008, issue 12, pp. 120-122.

[13] Wang Weshu, Zhou Yijun, Wu Haibo, Hui Chenyi, Jiao Yuguo. "Studies on new evaluation methods of organic chemistry experiment course for non-chemistry majoring students in university for nationalities”. Laboratory Science, 2013, Vol.16, issue 6, pp. 122-127.

[14] Li Yuxian, Zhu Lilin. "Reform and practice of organic chemistry experiment examination method of Chinese medicine professional". Forum on Contemporary Education, 2011, issue 11, pp. 54-56.
[15] Li Jianjun, Li Guoan. "The significance and ways of experimental process assessment”. Laboratory research and exploration, 2007, Vol.26, issue 4, pp.106-108.

[16] Bei Zhanyu, Luo Xiaobin, Li Jialin.”Research and practice of organic chemistry experiment examination methods of pharmaceutical professional”. Forum on Contemporary Education, 2008, Vol.27, issue 10, pp. 132-135.

[17] Yuan Chunlan, Ren Lijun. "Exploring the new methods on based teaching of the physicsl chemistry experiment”. Guangdong Chemical, 2013, Vol. 40, issue 255, pp.252-253,242.

[18] Li guoxiang, Li songbo. "Study and exploration of open teaching mode of basic chemistry experiment”. Inner Mongolia petrochemical industry, 2007, issue 1, pp. 21-22.

[19] Xu Yaqin, Fu Ying. "Research and exploration of open chemistry experiments”. Experimental technology and management, 2007, issue 2, pp. 14-15.

[20] Yang Liuqing. "Thoughts about integrating the scientific research progress into the teaching of basic chemistry experiment". The science educ, 2012, issue 3, pp. 123-124. 